\title{
Forced Response of an Electromagnetic Harmonic Movable Tooth Drive System to Current Excitation
}

\author{
Yongli Liang and Lizhong $\mathrm{Xu}^{*}$ \\ Mechanical Engineering Institute, Yanshan University, Qinhuangdao, 066004, China
}

\begin{abstract}
In this study, the authors proposed an electromagnetic harmonic movable tooth drive system., The forced response equation of the drive system to exciting currents was developed. Using this equation, the forced responses of the drive system to exciting currents were investigated. The results show that the forced responses of the drive system to the exciting currents were affected by mechanical and electric parameters. To reduce the vibrating amplitudes, smaller coil current, flexible ring radius, and average static displacement of the flexible ring, increased thickness of the flexible ring, clearance between the flexible ring and coils, and coil pole pair number should be taken into account. The results can be used for designing the system and further study of the dynamics performance of the drive system.
\end{abstract}

Keywords: Current excitation, electromagnetic drive, forced responses, harmonic drive, movable tooth drive.

\section{INTRODUCTION}

The electromagnetic harmonic drive can be used in the technical fields such as aviation and space flight. [1]. For years, the harmonic drives have been developed [2-4]. Janes investigated the method for avoiding eddy current through the metal flexible ring [5]. The controlling of high performance servo driving system for magnetic type harmonic gear drive was realized by means of software [6]. A novel magnetic harmonic gear drive with high reliability, inherent overload protection and high efficiency was proposed [7]. A control method of the harmonic magnetic gear to attenuate adverse effects due to cogging torque on the vibratory system was investigated [8].

In other words, a number of studies about the harmonic drives were conducted. The electromagnetic harmonic drive has a small output torque but the output torque of the mechanical harmonic drive is large, therefore the teeth had to be produced on the flexible ring which is a difficult task. Hence, the authors proposed an electromagnetic harmonic movable tooth drive system in which the teeth are removed from the flexible ring.

The proposed electromagnetic harmonic movable tooth drive system is shown in Fig. (1), which consists of three main parts: electromagnetic coils, harmonic movable tooth drive without wave generator, and flexible ring between the coils and the drive.

The coils are energized sequentially by currents which can result in generating a rotating electromagnetic field causing periodic elastic deformation of the flexible ring accompanied by periodic contact between the flexible.

Compared with other harmonic drives, the drive system has the main advantages as below: (1) rolling contact,

*Address correspondence to this author at the Mechanical Engineering Institute, Yanshan University, Hebei Street, Qinhuangdao, Postcard: 066004, China; Tel: 0335-8057031; E-mail: xlz@ysu.edu.cn transmitting load by meshing, and reduction; (2) high operating efficiency; and (3) small size.
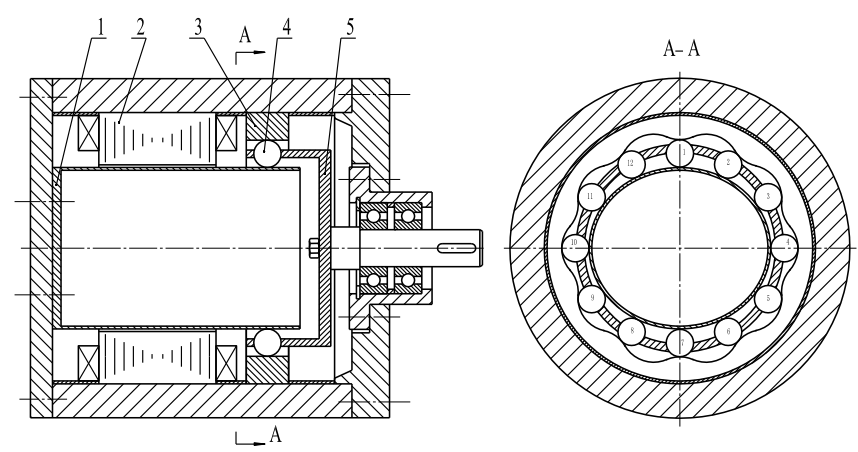

Fig. (1). An electromagnetic harmonic movable tooth drive system. 1. flexible ring; 2 . coils; 3 . rigid gear; 4. movable tooth; 5 . Rotor ring and the movable teeth. It causes the relative motion between the movable teeth and rigid ring. As the rigid ring is fixed, the meshing force drives the rotor to rotate.

In the drive system, the harmonic currents are generated which can cause undesirable forced responses of the drive system and result in noise and unacceptable performance characteristics. The prediction of the forced responses of the drive system to the exciting currents is required in the design stage for a drive system.

In this paper, for the proposed electromagnetic harmonic movable tooth drive system, the forced response equation of the drive system to exciting currents is presented. From the equation, the electromechanical coupled forced responses were analyzed. The results show that the forced responses of the drive system to the exciting currents were affected by mechanical and electric parameters. The results can be used in the design and for further study of the dynamics performance for the drive system. 


\section{ELECTROMECHANICAL COUPLED DYNAMICS EQUATION}

The dynamic equation of the flexible ring for the drive system is

$\frac{\partial^{4} \Delta w}{\partial \theta^{4}}+2 \frac{\partial^{2} \Delta w}{\partial \theta^{2}}+\Delta w=\frac{R_{m}{ }^{4}}{E I_{x}} \Delta q_{r}-\frac{R_{m}{ }^{4} \rho A}{E I_{x}} \frac{\partial^{2} \Delta w}{\partial t^{2}}$

Where $\Delta w$ is the dynamic displacement of the flexible ring, $R_{\mathrm{m}}$ is the average radius of the flexible ring, $I_{x}$ is the section modular of the ring, $\rho$ is the material density of the ring, $A$ is its cross section area, $t$ is the time, and $\Delta q_{r}$ is the dynamic electromagnetic force per unit arc length on the flexible ring.

The radial electromagnetic force per unit area on the flexible ring is:

$$
F_{d c}=\frac{B_{\delta}{ }^{2}}{2 \mu_{0}}=\frac{B_{0}{ }^{2}}{2 \mu_{0}\left(\delta_{0}-w\right)^{2}}
$$

Where, $\quad B_{0}=\frac{\mu_{0} F_{1}}{\delta_{0}}=1.35 \frac{\mu_{0} N I}{\delta_{0} p} ; \mu_{0}$ is the magnetic conductivity of free space, $\mu_{0}=4 \pi \times 10^{-7} \mathrm{~W} / \mathrm{m}$.A; $N$ is the coil number; $I$ is the coil current; $\delta_{0}$ is the initial gas gap; and $p$ is the pole pair number.

From Eq. (2), the dynamic electromagnetic force can be calculated as:

$\Delta F_{d c}=\frac{\mu_{0} N_{k}^{2} I^{2}}{p^{2}\left(\delta_{0}-w\right)^{3}} \Delta w-\frac{\mu_{0} N_{k}^{2} I}{p^{2}\left(\delta_{0}-w\right)^{2}} \Delta I_{i s}$

When the current is stable, letting $\Delta I_{s}=0$,.Eq.(3) may be changed into:

$\Delta q_{r 1}=\frac{d F_{d c}}{d w} \Delta w=\frac{B_{0}^{2} \delta_{0}^{2}}{\mu_{0}\left(\delta_{0}-w\right)^{3}} \Delta w$

Substituting Eq. (4) into Eq. (1), yields

$$
\begin{aligned}
\frac{\partial^{4} \Delta w}{\partial \theta^{4}} & +2 \frac{\partial^{2} \Delta w}{\partial \theta^{2}}+\Delta w \\
& =\frac{R_{m}{ }^{4} B_{0}{ }^{2} \delta_{0}{ }^{2}}{E I_{x} \mu_{0}\left(\delta_{0}-w\right)^{3}} \Delta w-\frac{R_{m}{ }^{4} \rho A}{E I_{x}} \frac{\partial^{2} \Delta w}{\partial t^{2}}
\end{aligned}
$$

Let $\Delta w=\phi(\theta) q(t)$, substituting it into (5), we obtain

$\phi^{(4)}(\theta)+2 \phi^{\prime \prime}(\theta)+Q \phi(\theta)=0$

Where,

$$
\begin{aligned}
& P=1-\frac{R_{m}{ }^{4} B_{0}{ }^{2} \delta_{0}{ }^{2}}{E I_{x} \mu_{0}\left(\delta_{0}-w\right)^{3}} \\
& Q=P-\frac{\rho A R_{m}{ }^{4}}{E I_{x}} \omega^{2}
\end{aligned}
$$

The general solution of Eq. (6) is

$\phi(\theta)=A_{1} \cos m_{1} \theta+A_{2} \sin m_{1} \theta+A_{3} \operatorname{ch} m_{2} \theta+A_{4} \operatorname{sh} m_{2} \theta$

At $\theta=0$ and $\theta=\frac{\pi}{2}$, rotating angle of normal, and shear force, i.e. $\phi^{\prime}(\theta)_{\theta=0}=0, \phi_{2}^{\prime}(\theta)_{=\frac{\pi}{2}}=0, \phi^{(3)}(\theta)_{\theta=0}=0$, and $\phi^{(3)}(\theta)_{\theta=\frac{\pi}{2}}=0$. Substituting Eq. (7) into the conditions, yields

$\left(m_{1} m_{2}{ }^{3}+m_{1}{ }^{3} m_{2}\right)^{2} \sin \left(\frac{\pi}{2} m_{1}\right) \operatorname{sh}\left(\frac{\pi}{2} m_{2}\right)=0$

From Eq. (8), the natural frequencies of the drive system can be obtained. Substituting the natural frequencies into Eq. (7), and let $A_{1}=1$, the mode function can be given by:

$$
\phi(\theta)=\cos m_{1} \theta+\frac{\left(m_{1}-m_{1}^{3}\right) \operatorname{sh}\left(\frac{\pi}{2} m_{1}\right)}{\left(m_{2}-m_{2}^{3}\right) \operatorname{sh}\left(\frac{\pi}{2} m_{2}\right)} c h m_{2} \theta
$$

\section{FORCED RESPONSES TO EXCITING CURRENTS}

If the exciting current is generated, dynamic electromagnetic force is produced. It causes forced response of the drive system. Letting the exciting current be $\Delta I_{i s}=E \sin \omega_{z} t$ (Here, $\omega_{z}$ is exciting frequency of the current, $E$ is its amplitude). From Eq.(3), the dynamic electromagnetic force can be given as:

$$
\Delta q_{r 2}=\frac{\mu_{0} N_{k}^{2} I}{p^{2}\left(\delta_{0}-w_{0}\right)^{2}} E \sin \omega_{z} t
$$

Substituting Eq. (10) into Eq. (1), the equation of the forced response of the drive system to the current excitation can be given as:

$\ddot{q}_{i}(t)+\omega_{i}^{2} q_{i}(t)=Q_{i}(t) \quad(i=1,2, \cdots)$

Where $I_{m d}=\frac{R_{m}{ }^{4} \mu_{0} N_{k}{ }^{2} I}{E I_{x} p^{2}\left(\delta_{0}-w_{0}\right)^{2}} I_{m}$

Using orthogonality, Eq. (11) can be changed into the following form:

$$
\ddot{q}_{i}(t)+\omega_{i}^{2} q_{i}(t)=Q_{i}(t) \quad(i=1,2, \cdots)
$$

Here, $Q_{i}(t)$ is the generalized force. It equals

$Q_{i}(t)=\int_{0}^{\frac{\pi}{2}} \Delta q_{r} \phi_{i}(\theta) r d \theta \quad i=(1,2, \cdots)$

$q_{i}(t)$ is the generalized coordinate, then

$q_{i}(t)=\frac{1}{\omega_{i}} \int_{0}^{t} Q_{i}(\tau) \sin \omega_{i}(t-\tau) d \tau \quad i=(1,2, \cdots)$ 

yields

Substituting Eqs. (9) and (10) into Eqs. (13) and (14),

$$
\begin{aligned}
Q_{i}(t)= & {\left[\frac{A_{1 i}}{m_{1 i}} \sin \left(m_{1 i} \frac{\pi}{2}\right)+\frac{A_{3 i}}{m_{2 i}} \operatorname{sh}\left(m_{2 i} \frac{\pi}{2}\right)\right] } \\
& \cdot \frac{\mu_{0} N_{k}^{2} I R_{m}}{p^{2}\left(\delta_{0}-w_{0}\right)^{2}} E \sin \omega_{z} t \\
q_{i}(t)=\frac{1}{\omega_{i}} & {\left[\frac{A_{1 i}}{m_{1 i}} \sin \left(m_{1 i} \frac{\pi}{2}\right)+\frac{A_{3 i}}{m_{2 i}} \operatorname{sh}\left(m_{2 i} \frac{\pi}{2}\right)\right] . } \\
& \frac{\mu_{0} N_{k}{ }^{2} I R_{m} E}{p^{2}\left(\delta_{0}-w_{0}\right)^{2}} \cdot \frac{1}{\omega_{z}{ }^{2}-\omega_{i}^{2}}\left(\omega_{i} \sin \omega_{z} t-\omega_{z} \sin \omega_{i} t\right)
\end{aligned}
$$

In Eq. (16), the first term represents the forced response of the drive system to current excitation, and the second is the associated free vibration, which vanishes soon under dampness and can be neglected. The dynamic displacement of the flexible ring can be expressed as,

$$
\Delta w(\theta, t)=\sum_{i=1}^{\infty} \phi_{i}(\theta) q_{i}(t)
$$

\section{RESULTS AND DISCUSSION}

Considering an electromagnetic harmonic movable tooth drive system defined by the data given in Table $\mathbf{1}$, the material used for the flexible ring was steel. It was subjected to an electromagnetic force. Equations (9), (16) and (17) were utilized for the analysis of the forced response of the flexible ring to an exciting current. The forced responses corresponding to each mode are given as shown in Fig. (2) where only the first four modes were considered. Here, the exciting current $E=1 \mathrm{~A}$, and exciting frequency $\omega_{z}=50 \mathrm{~Hz} / \mathrm{s}$. Figs. (3, 4) show the effects of the electromechanical parameters on the forced responses. From Fig. (2), it is shown that:

Table 1. Parameters of drive system.

\begin{tabular}{|l|c|}
\hline outer radius of flexible ring $R(\mathrm{~mm})$ & 39 \\
\hline inter radius of rotor $R_{\mathrm{n}}(\mathrm{mm})$ & 40 \\
\hline thickness of flexible ring $\tau(\mathrm{mm})$ & 0.2 \\
\hline length of flexible ring $l(\mathrm{~mm})$ & 140 \\
\hline coil number $n$ & 81 \\
\hline Pole pair number $p$ & 3 \\
\hline
\end{tabular}

Under simple harmonic current excitation, the forced responses of the flexible ring for the drive system are simple harmonic vibration. At different points of the flexible ring, the amplitudes of the vibrations change periodically with the time. But, the relative amplitude relationship between the different points of the flexible ring was constant for each mode.

For mode 1, peak displacements occur at positions $\theta=0^{\circ}, \theta=90^{\circ}$ and $\theta=180^{\circ}$. For mode 2, the peak displacements occur at positions $\theta=0^{\circ}, 45^{\circ}, 90^{\circ}$, $135^{\circ}$, and $180^{\circ}$. For mode 3 , the peak dynamic displacements occur at positions $\theta=0^{\circ}, 30^{\circ}, 60^{\circ}, 90^{\circ}$, $120^{\circ}, 150^{\circ}$, and $180^{\circ}$. For mode 4 , five positive peak displacements and four negative peak displacements occur.

As the exciting current frequency is similar to the natural frequency of the mode 1 , the forced response amplitude for the mode 1 is the largest, and the total forced response is close to one for the mode 1 .

From Fig. (3), it is known:

As the coil current $I$ increases, the natural frequencies of the drive system decrease and the vibrating amplitudes of the flexible ring to the current excitation increase because the electromagnetic force affects the flexibility of the flexible ring.

(2) As the thickness $\tau$ of the flexible ring increases, the natural frequency of the drive system increases and the vibrating amplitudes of the flexible ring to the current excitation decrease because the increase in the thickness causes stiffness in the flexible ring.

As the clearance $\delta_{0}$ between the flexible ring and coils increases, the natural frequency of the drive system increases and the vibrating amplitudes of the flexible ring to the current excitation decrease because a large clearance $\delta_{0}$ between the flexible ring and coils can cause decrease of the electromagnetic force for a given coil current and the stiffness increase of the flexible ring.

(4) As flexible ring radius $R_{\mathrm{m}}$ increases, the natural frequency of the drive system decreases and the vibrating amplitudes of the flexible ring to the current excitation increase.

As the average static displacement $w_{0}$ of the flexible ring increases, the natural frequency of the system decreases and the vibrating amplitudes of the flexible ring to the current excitation increase. It is because a large static displacement $w_{0}$ can cause increase in the electromagnetic force for a given coil current.

As the number of coil pole pair increases, the natural frequency of the system also increases and the vibrating amplitudes of the flexible ring to the current excitation decrease.

As the order number of the vibrating modes increases, the effects of these parameters on the forced responses change. For mode 2, the vibrating amplitudes of the flexible ring to the current excitation decrease more obviously with decreasing the currents. The vibrating amplitudes of the flexible ring increase more slowly with decreasing the thickness $\tau$ of the flexible ring. The vibrating amplitudes of the flexible ring increase more obviously with decreasing the clearance $\delta_{0}$ between the flexible ring and coils. The vibrating amplitudes of the flexible ring increase more slowly with increasing the flexible ring radius $R_{\mathrm{m}}$. 
(a) mode 1

model

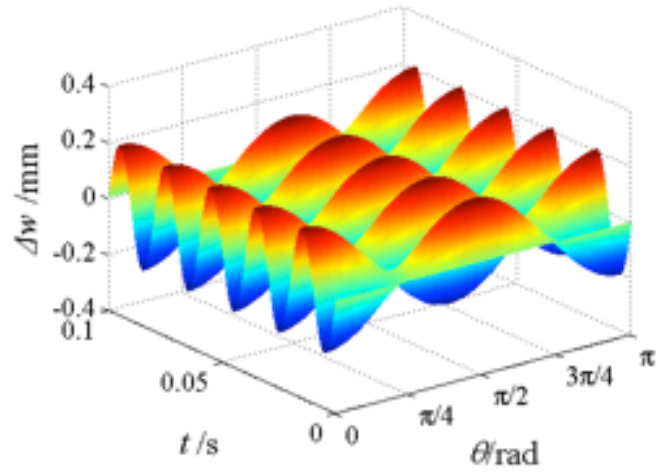

(b) mode 2

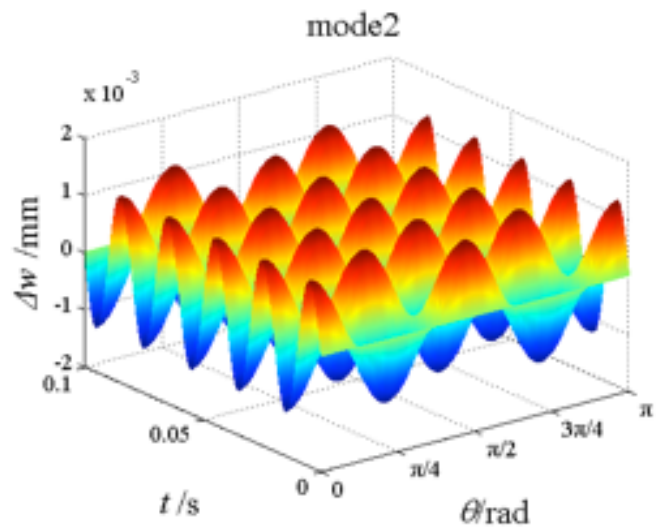

(c) mode 3

mode3

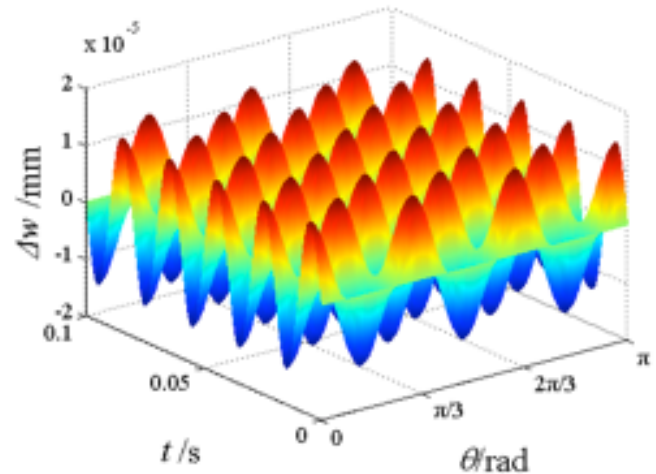

(d) mode 4

mode 4

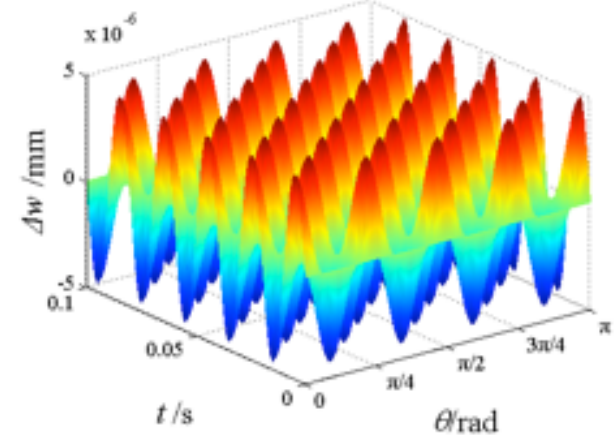

Fig. (2). Forced responses of the flexible ring to exciting current. (a) I changes

mode1

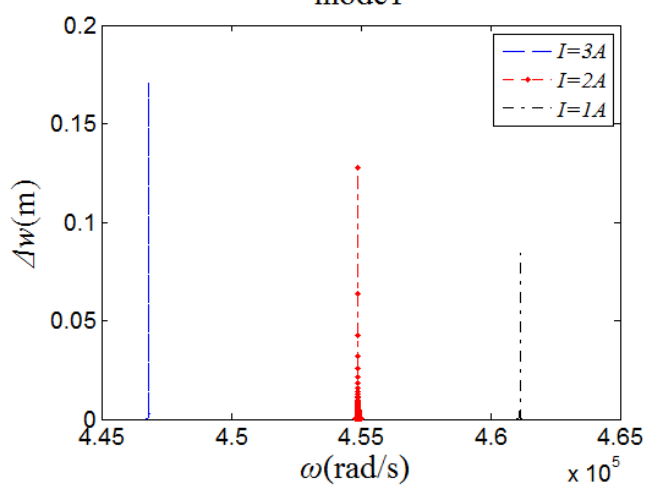

(b) $\tau$ changes

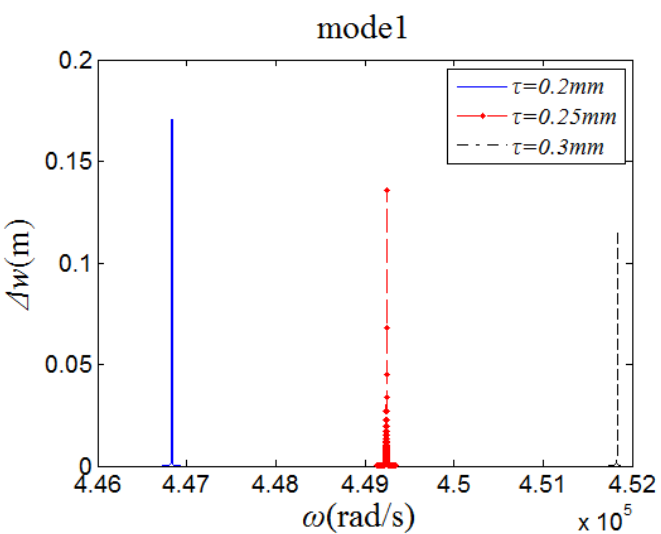

(c) $\delta_{0}$ changes

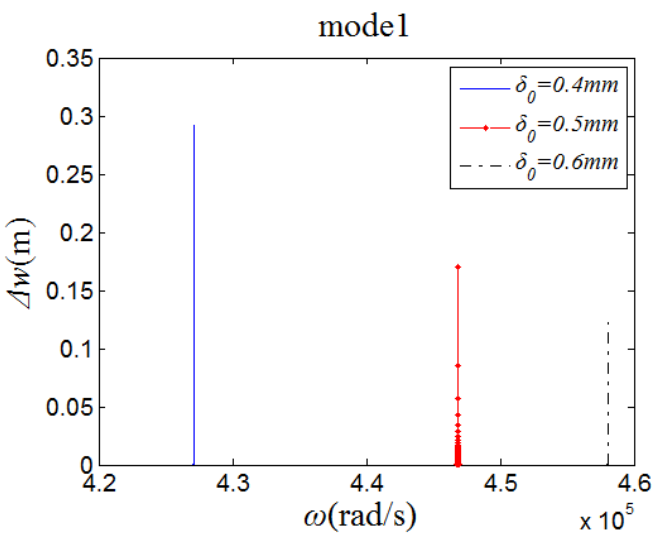

(d) $R_{\mathrm{m}}$ changes

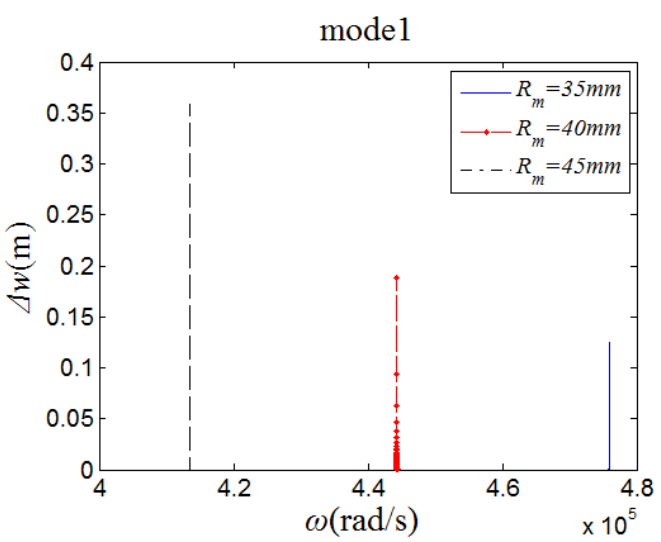


(e) $w_{0}$ changes

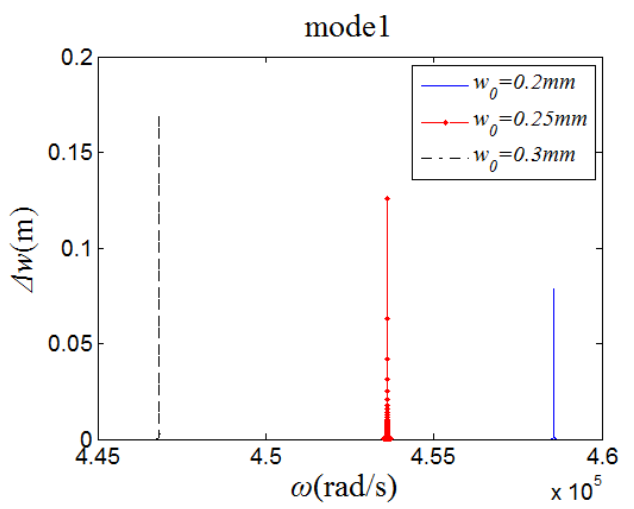

(f) $p$ changes

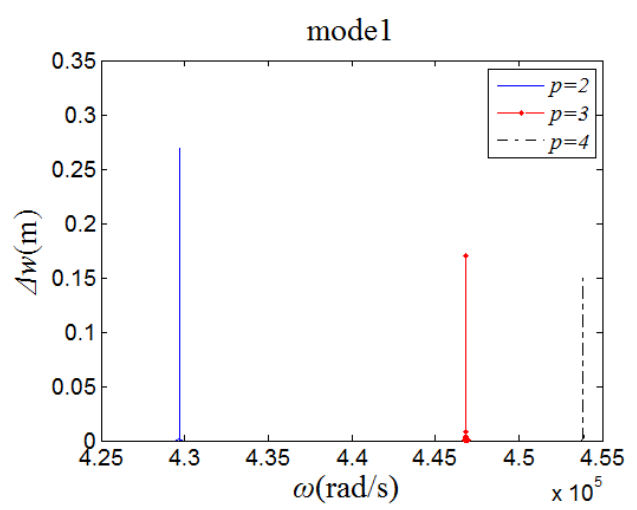

Fig. (3). Effects of the electromechanical parameters on the forced responses (mode 1).

(a) I changes

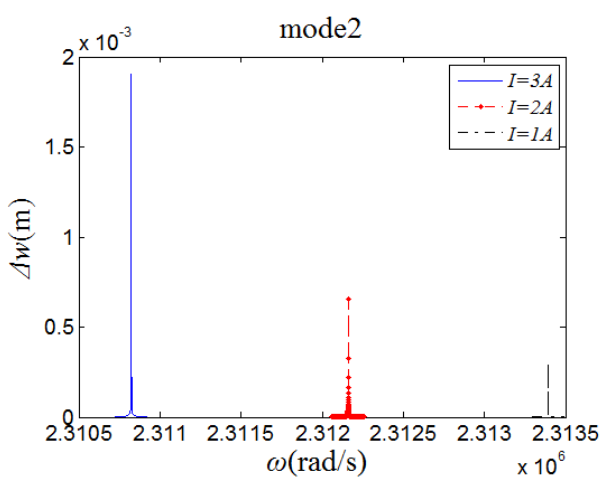

(b) $\tau$ changes

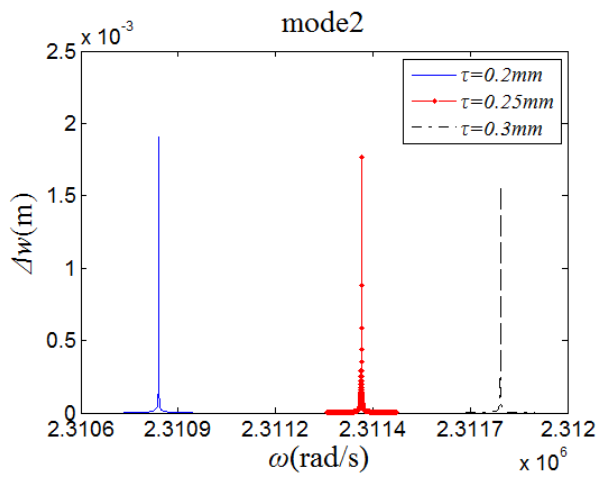

(c) $\delta_{0}$ changes

(Fig. 4) contd.....

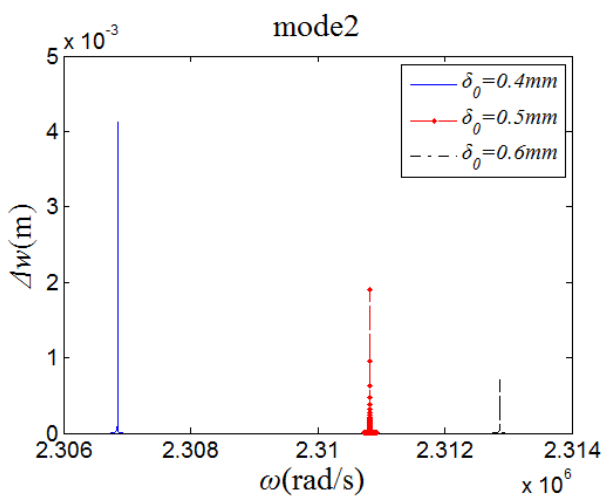

(d) $R_{\mathrm{m}}$ changes

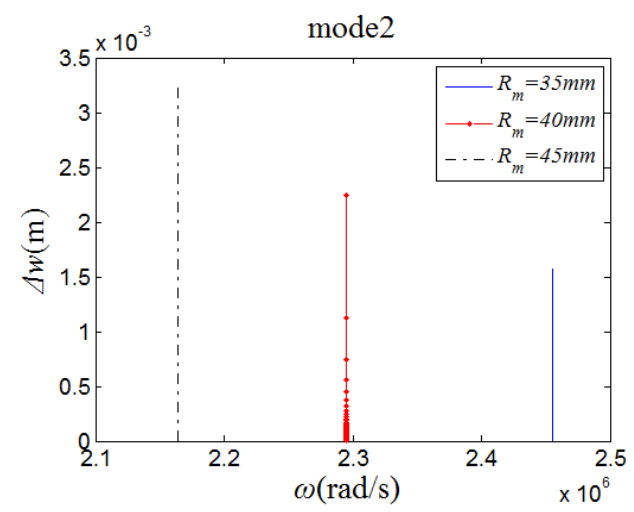

Fig. (4). Effects of the electromechanical parameters on the forced responses (mode 2).

In other words, the vibrating amplitudes of the flexible ring for the drive system are affected by mechanical and electric parameters. To reduce the vibrating amplitudes, smaller coil current $I$, increased thickness $\tau$ of the flexible ring, increased clearance $\delta_{0}$ between the flexible ring and coils, smaller flexible ring radius $R_{\mathrm{m}}$, smaller average static displacement $w_{0}$ of the flexible ring, and larger coil pole pair number should be taken into account. Certainly, the output torque of the drive system will decrease when the coil current $I$, and the flexible ring radius $R_{\mathrm{m}}$ are reduced, or the clearance $\delta_{0}$ between the flexible ring and coils is increased. Therefore, these parameters should be selected properly.

\section{CONCLUSION}

In this paper, for the proposed electromagnetic harmonic movable tooth drive system, the forced responses of the drive system to exciting currents were analyzed. The results show that the forced responses of the drive system to the exciting currents are affected by mechanical and electric parameters. To reduce the vibrating amplitudes, smaller coil current, flexible ring radius, average static displacement of the flexible ring, increased thickness of the flexible ring, clearance between the flexible ring and coils, and coil pole pair number were observed .

\section{CONFLICT OF INTEREST}

The authors confirm that this article content has no conflict of interest. 


\section{ACKNOWLEDGEMENTS}

This project is supported by National Natural Science Foundation of China (No.51275441).

\section{REFERENCES}

[1] D. F. Herdeg, Electromagnet Harmonic Drive Low Inertia Serve, Patent AD442879, Dec 1963.

[2] W. Trimmer, and R. Jebens, "Harmonic electrostatic motors", Sensors and Actuators, vol. 20, pp. 17-24, 1989.

[3] O. Barth, "Harmonic piezodrive-miniaturized servo motor", Mechatronics, vol. 10, pp. 545-554, 2000.
[4] O. Kayabasi, and F. Erzincanli, "Shape optimization of tooth profile of a flexspline for a harmonic drive by finite element modeling", Materials \& Design, vol. 28, pp. 441-447, 2007.

[5] C. F. Janes, "Electro-Magnetic Actuation", U.S. Patent 3200668, Aug 1965.

[6] Z. Shang, and Z. You, "Control system for magnetic type harmonic gear drive", Manufacturing Technology Machine Tool, vol. 3, pp. 7-10, 1997 (in China)

[7] J. Rens, K. Atallah, S.D. Calverley, and D. Howe, "A novel magnetic harmonic gear", In: IEEE Transactions on Industry Applications, vol. 46, pp. 206-212, 2010.

[8] U. Yutaka and S. Yasushi, "Cyclic disturbance suppression control for harmonic magnetic gear", In: IEEJ Transactions on Industry Applications, vol. 134, pp. 284-292, 2014.

(C) Liang and $\mathrm{Xu}$; Licensee Bentham Open

This is an open access article licensed under the terms of the Creative Commons Attribution Non-Commercial License (http://creativecommons.org/licenses/by-nc/4.0/) which permits unrestricted, non-commercial use, distribution and reproduction in any medium, provided the work is properly cited. 